\title{
Increased filtration efficiency of attached compared to free-swimming flagellates
}

\author{
Karen K. Christensen-Dalsgaard, Tom Fenchel*
}

Marine Biological Laboratory, University of Copenhagen, Strandpromenaden 5, 3000 Helsingør, Denmark

\begin{abstract}
Heterotrophic flagellates often attach to surfaces while feeding. A possible fluid dynamic reason is that attachment increases filtration efficiency. This was investigated experimentally and theoretically. The effect of attachment on flow field and clearance was studied by video microscopy in the nanoflagellates Paraphysomonas vestita and Pteridomonas danica. The flow field around feeding cells was visualised by latex bead suspensions. From a fluid dynamics point of view, it is advantageous to be sessile if the radius of the transectional area of the water flow that is swept for particles is smaller than $1.5 \times$ cell radius. For flagellates with a smooth flagellum, this also requires that the flagellum beats with a large amplitude. For attached $P$. vestita, clearance is ca. $70 \%$ higher relative to free-swimming cells and for $P$. danica this value is ca. $34 \%$. The difference between the 2 species accords with the fact that Pteridomonas sweeps a wider area of the water flow relative to its cell size than Paraphysomonas. Protozoa may attach for other reasons, such as to remain at sites with high food concentrations, but in the case of suspension feeders, it is also advantageous to attach in terms of fluid dynamics. It is likely that a large proportion of the heterotrophic nanoflagellates in plankton are associated with suspended particles that act as drift anchors, thus enhancing clearance.
\end{abstract}

KEY WORDS: Flagellates · Suspension feeding $\cdot$ Microbial loop $\cdot$ Filtration efficiency $\cdot$ Attachment · Fluid dynamics Resale or republication not permitted without written consent of the publisher

\section{INTRODUCTION}

Heterothrophic flagellates are important components of the microbial food web. Bacteria are essential for the re-utilisation of decomposed organic matter, including ca. $25 \%$ of the planktonic primary production estimated to occur as exudates (e.g. Larsson \& Hagström 1979). Heterotrophic flagellates play an important ecological role by forming a link between bacteria and larger suspension feeders; in Limfjorden, Denmark, ca. $20 \%$ of the water is cleared for bacteria by flagellates per day (Fenchel 1982c). Although there is a considerable variation in the occurrence of flagellates, the same order of magnitude seems valid for many other aquatic habitats (Fenchel 1986a). Thus, improved knowledge on feeding processes of these organisms could lead to a deeper understanding of the processes involved in the transfer of carbon in marine food webs.
Some flagellate species strain food particles from the water with a filtration apparatus in the form of an array of radial pseudopodia or tentacles. In other species, suspended food particles are intercepted directly by a certain part of the cell surface or by the flagellum. The water flow results from swimming through the water, or - if the cells are attached-by generating feeding currents. In both cases this results from propagation of planar or 3D waves along the flagellum. In smooth flagella this is mediated by a force acting on the fluid, generated by the flagellum itself. If the flagellum is hispid and has rigid projections (mastigonemes) protruding from its side, the force against the fluid is generated by the mastigonemes (Brennen 1976). Most flagellates (and other suspension-feeding protists) tend to attach to particles or surfaces while feeding, and so attachment must be assumed to be important in the feeding process. 
An obvious reason for attachment is to remain in areas with a high food concentration. Motile bacteria make up at least $20 \%$ of marine planktonic bacterial populations (Fenchel 2001), and it has long been known that motile bacteria exhibit chemotactic responses to chemical gradients (Weilbull 1960, Adler 1969). This leads to aggregation of bacteria around sources of dissolved organic carbon (e.g. Bell \& Mitchell 1972, Brown et al. 1993), and thus a patchy distribution of bacteria in the water column. Large suspended particles tend to harbour high concentrations of bacteria; the concentration in association with marine snow particles is typically $10^{2}$ to $10^{5}$ times higher than that of the surrounding water (Alldredge \& Silver 1988). Protozoa are likewise capable of motile responses to chemical stimuli, and aggregate around patches with high food concentration (Fenchel \& Blackburn 1999). Further attachment to, e.g., suspended particles ensures that the protozoa remain in such hotspots of food particles.

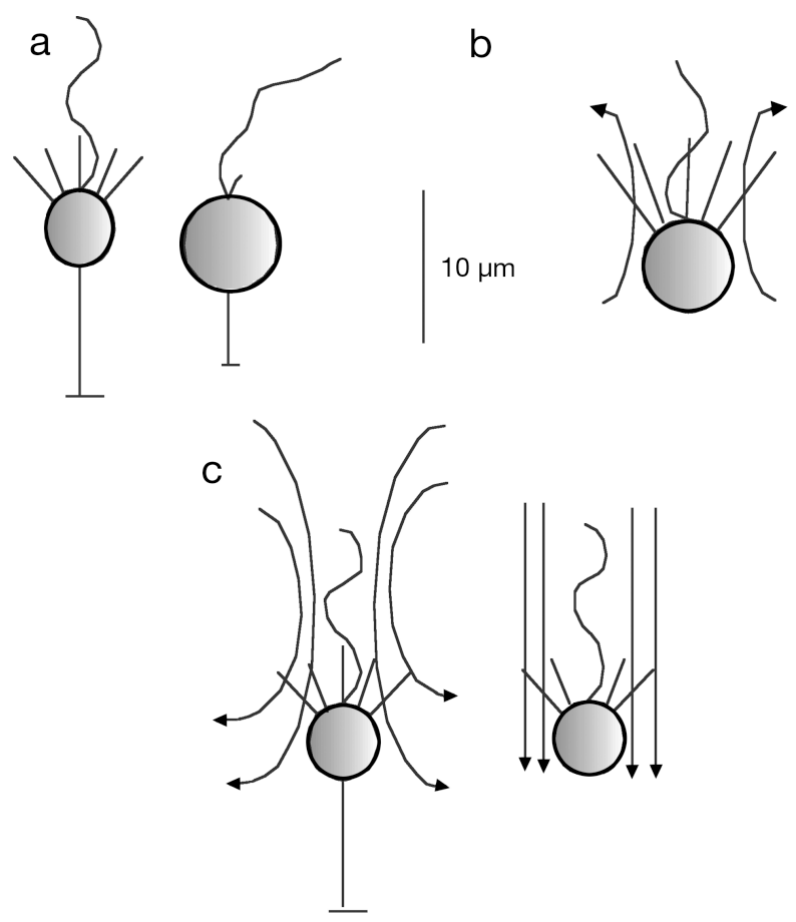

Fig. 1. (a) Schematic drawings of Pteridomonas danica (left) and Paraphysomonas vestita. (b) Schematic drawing of a choanoflagellate; these have a smooth flagellum so that the direction of water flow is parallel to the wave direction of the flagellum. (c) Schematic presentation of the differences in the flow field of an attached and a free-swimming flagellate relative to the cell. In the former case, water is accelerated towards the middle of the flagellum. In the latter case, practically no movement of the water is induced except for a slight lateral displacement of water around the translating cell. The cell then only experiences a parallel flow with an opposite direction, but similar magnitude as swimming velocity
This could be one reason to attach in the presence of high densities of food bacteria. But the fact that protozoa tend to attach to solid surfaces, irrespective of the presence of chemical cues, implies that other reasons must be important. Starving cells do not attach, but tend to swim continuously (Fenchel 1982a), suggesting that swimming largely serves to find more suitable habitats rather than for feeding.

A possible hydrodynamic reason for attachment is investigated in this paper. When a flagellate is attached to a particle, the drag of the particle is balanced by thrust produced by the flagellum. This generates a water current with direction towards the cell body and opposing the swimming direction. If cells attach to a particle that can be dragged along, this reduces swimming velocity, but the total fluid velocity experienced by the organism may still be higher because of the flow drawn towards the cell. If the particle is so large that the flagellate cannot drag it, there is no forward motion and the entire force produced by the flagellum is used to generate thrust. The flow field experienced by free-swimming flagellates is different from that experienced if it is attached, even when the parameters of flagellar motion remain the same (Fig. 1).

In the present study, general fluid dynamic effects of attachment are discussed theoretically, emphasising flagellates with a single smooth flagellum such as choanoflagellates. This is possible because the theory of smooth flagella has been extensively studied (e.g. Hancock 1953, Gray \& Hancock 1955, Lighthill 1975, 1976). The hydrodynamics of hispid flagella have not been adequately studied from a theoretical point of view, so it was not possible to provide a precise analysis of the fluid dynamic effects of attachment specific for these organisms. Instead, experiments were carried out on 2 ecologically important flagellates, Paraphysomonas vestita and Pteridomonas danica, to determine the effect on feeding efficiency of attachment to particles of various sizes, compared to the free-swimming case. Both of these flagellates have hispid flagella, but very different flagellar beat patterns (ChristensenDalsgaard \& Fenchel unpubl.).

In marine systems, flagellates with smooth flagella are primarily represented by choanoflagellates. Most species are permanently attached by a stalk (only propagules swim). The free-swimming, acanthoecid choanoflagellates build a large and complex lorica. This lorica acts as a drift anchor in a manner similar to the situation when other types of flagellates attach to suspended particles. For this reason, we could not compare the effect on clearance between freeswimming and attached cells experimentally for choanoflagellates. Thus the situation for flagellates with smooth flagella was treated only theoretically. 


\section{MATERIALS AND METHODS}

General information. Batch cultures of Paraphysomonas vestita and Pteridomonas danica were grown to densities of about $10^{4}$ cells ml $\mathrm{m}^{-1}$ in boiled and filtered seawater (salinity 20\%o), with a suspension of an unidentified bacterium (ca. $10^{8}$ cells $\mathrm{ml}^{-1}$ ). Schematic drawings of $P$. danica and $P$. vestita are shown in Fig. 1. Both are ubiquitous in seawater, including the water column as well as sediments. The mean size of the latter species is $5.7 \mu \mathrm{m}$, and that of the former is $4.9 \mu \mathrm{m}$. $P$. danica carries a conical array of tentacles around the flagellum that intercepts suspended bacteria. $P$. vestita has 2 flagella, a short and a long one; the latter is responsible for the generation of water flow. Suspended particles that touch the flagellum (or the mastigonemes) are transported to the mouth, which is situated beneath the base of the small flagellum. Both species are capable of attaching to solid surfaces with a mucous thread secreted from the posterior pole of the cell. All measurements of cell diameters, flagellar lengths, etc., were based on video recordings.

Experiments were conducted at room temperature $\left(20\right.$ to $25^{\circ} \mathrm{C}$ ). As this is higher than most ambient seawater temperatures, control experiments were conducted at $6^{\circ} \mathrm{C}$. At this temperature (after $48 \mathrm{~h}$ incubation), flow velocities were reduced by only $7 \%$ for Paraphysomonas vestita and $11 \%$ for Pteridomonas danica compared to room temperature, and so the results of the present study also apply to natural habitats at lower ambient temperatures.

Recordings of water flow, swimming, and attachment were based on cell suspensions in an 'O-ring chamber'. An O-ring (height: $2 \mathrm{~mm}$, diameter: $15 \mathrm{~mm}$ ) was attached to a microscope slide with vaseline, and flagellate suspensions were applied inside the ring with a pipette. The O-ring was then covered with a cover slip, avoiding air bubbles, providing an observation chamber with a volume of $0.35 \mathrm{ml}$. When nothing else is stated, observations and recordings were carried out using dark-field illumination and a $20 \times$ objective.

Attachment rates of swimming cells were also studied in an O-ring chamber. After filling the O-ring with a cell suspension, numbers of attached cells within an area $(800 \times 800 \mu \mathrm{m})$ of the cover slips were counted at 2 min intervals. Tests were made with both starved and feeding cells placed either in bacterial suspension or in particle-free water.

Video recordings. Video recordings were made to track flows and estimate flow velocities around feeding cells, both when attached and when swimming. In order to visualise flow fields, suspensions of latex beads were used. Suspensions were prepared by adding $0.2 \mu \mathrm{m}$ particles to a final concentration of about $10^{10}$ particles $\mathrm{ml}^{-1}$. Particles were first suspended in demineralised water and then the flagellate suspension was slowly added. This was done in order to avoid aggregation of the particles. The $0.2 \mu \mathrm{m}$ particles are not retained by the flagellates, nor do they in any way interfere with their behaviour. They can therefore be considered as neutral indicators of water flow.

Video recordings were made with an $\mathrm{EDH}^{\odot} \mathrm{KamPro}$ 02 CCD camera and a Sony digital video recorder at a frequency of $25 \mathrm{~Hz}$, giving a temporal resolution of $0.04 \mathrm{~s}$. Frame-by-frame tracking of the currents was subsequently done, either computationally using the program LabTrack QT (DiMedia), or manually. For manual tracking, the position of cells and particles was plotted directly from the screen to a transparent overlay attached to the screen during frame-by-frame playback of the recordings.

Flow was investigated under the following circumstances: (1) Free-swimming cells and cells dragging particles. Latex spheres (diameter $25 \mu \mathrm{m}$ ) or cornstarch were added to the cultures $24 \mathrm{~h}$ prior to recording. Both flagellate species were found to attach to cornstarch particles, which had diameters typically ranging between 5 and $15 \mu \mathrm{m}$, but only Paraphysomonas vestita attached to latex spheres. During the recordings, cells were swimming freely or attached to and pulling one or more particles. Altogether, 61 recordings were made of $P$. vestita, and 112 recordings of Pteridomonas danica. (2) $P$. vestita attached to the surface of the microscope slide and generating a current parallel to the slide (Fenchel 1986b). Altogether, 10 recordings were analysed. (3) Cells attached to large agar spheres. The agar spheres were made by dropping a warm solution of India ink and seawater (salinity $20 \%$ ) with $2 \%$ agar into paraffin oil overlying sterile seawater (Fenchel 2001). The India ink was added to make it possible to use the spheres in darkfield illumination without generating too much scattered light. The spheres measured 600 to $1000 \mu \mathrm{m}$ in diameter. Recordings were made of cells attached halfway up to the surface of the sphere, generating currents perpendicular to the surface. In all, 34 recordings were made of $P$. vestita and 35 of $P$. danica.

The velocity of the water that can be strained for food particles is of particular interest. It was quantified immediately ahead of the cells at the level of the midpoint of the flagellum. For species with a filter, the transectional area of relevance, $A$ (where $R$ is the radius of this area), is that of the aperture of the filter. Paraphysomonas does not have a filter, but the area circumscribed by flow lines that just allows particle encounter with the flagellum (the 'filtration disk') is approximately equal to the projected area of the cell ( $R=a$, where $a$ is the radius of the cell). Measured 
quantities and other symbols used in the following text are summarised in Table 1.

Force generated by the flagellum. Video recordings using a $10 \times$ objective were made of Paraphysomonas vestita pulling latex spheres with a diameter of $25 \mu \mathrm{m}$ in otherwise particle-free water, in order to calculate the force generated by the flagellum. As Pteridomonas danica did not attach to the latex balls, it was only possible to do these calculations on $P$. vestita. The height of the sphere above the microscope slide was measured, and only cells swimming at distances greater than $125 \mu \mathrm{m}$ from the nearest surface were recorded. This was done to avoid interference from wall effects; these should be negligible at distances to the surface greater than 10 times the radius of the latex sphere (Happel \& Brenner 1973). In total, 15 recordings were made.

\section{RESULTS}

\section{Theoretical considerations on the fluid dynamics of attachment}

Situations that involve generation of thrust as well as swimming are difficult to analyse. Here we limit our considerations to the 2 extreme cases: (1) where only negligible thrust and motion of the water are produced, so that all force is used for forward motion (zero thrust swimming); and (2) where there is no forward motion, and so the entire force is used in generating thrust and a large-scale flow field.

In Case (1), the flow of the fluid experienced by the organism is uniform everywhere, and with a velocity of equal magnitude to, but in opposite direction of, the swimming velocity (ignoring a slight lateral displacement around the cell as it translates through the water). The flow $Q\left(\mathrm{~L}^{3} \mathrm{~T}^{-1}\right)$ through a filtration disk with radius $R$ perpendicular to the swimming direction can be calculated from:

$$
Q=\pi R^{2} U
$$

where $U$ is the swimming velocity. If the organism is considered to be spherical with radius $a_{\text {, the swimming }}$ speed is given by Stokes' equation as:

$$
U=\frac{F}{6 \pi \eta a}
$$

where $\eta$ is the viscosity of the fluid $\left(10^{-3} \mathrm{Ns} \mathrm{m}^{-2}\right)$, and $F$ the force generated by the flagellum. This corresponds to:

$$
Q=\frac{F R^{2}}{6 \eta a}
$$

In Case (2), the thrust generates a velocity gradient and thus a pressure gradient in the fluid. This results in a more complicated flow pattern around the organism. If we assume that the force is a point force in an unbounded fluid, and that the cell body does not interfere significantly with the flow, the velocity field of the fluid can be modelled by singularity analysis (e.g. Lighthill 1975):

$$
U_{r}=\frac{F \cos \theta}{4 \pi r \eta} \quad U_{\theta}=\frac{-F \sin \theta}{8 \pi r \eta}
$$

where the position in the fluid relative to the force is given in polar coordinates $(r, \theta) ; U_{\mathrm{r}}$ is the radial component of the velocity and $U_{\theta}$ the transverse velocity component. The resultant flow field can be seen in Fig. 2. The velocity component relevant for the feeding cell is the one parallel to the cellular axis through the filtration disk. By integration over the radial velocity gradient (Fenchel 1986b), the flow through the filtration area, if positioned at the centre of the flagellum, is found to be:

$$
Q=\frac{F R}{4 \eta}
$$

\begin{tabular}{|c|c|}
\hline Abbreviation & Definition \\
\hline a & Cell radius \\
\hline 1 & Length of undulating flagellum from base to tip ( $=N \lambda$ where $N$ is number of waves and $\lambda$ is wave length) \\
\hline$L$ & Stretched length of flagellum \\
\hline$R$ & Radius of transectional area $A$ of flow in front of the cell (filtration disk) within which particles are intercepted \\
\hline$R^{*}$ & Maximum radius of $R$ allowing for increasing flow when attached \\
\hline$Q$ & Flow through $A$ \\
\hline$V_{\mathrm{t}}$ & Total velocity through $A$ \\
\hline$V_{\mathrm{s}}$ & Component of $V_{\mathrm{t}}$ caused by swimming \\
\hline$V_{\mathrm{c}}$ & Component of $V_{\mathrm{t}}$ caused by drag \\
\hline$\dot{U}$ & Velocity \\
\hline$F$ & Force \\
\hline$\eta\left(10^{-3} \mathrm{~N} \mathrm{~s} \mathrm{~m}^{-2}\right)$ & Viscosity \\
\hline
\end{tabular}

It can easily be seen, comparing Eqs. (2) \& (4), that the velocity of the fluid at the centre of the flow field

Table 1. Definitions of symbols throughout text and in equations 
will be of larger magnitude than the velocity with which the same force can pull a cell of any given size. However, in Case (1), $Q$ is directly proportional to the filter area, and thus to $R^{2}$. In Case (2), because the magnitude of the velocity of the fluid decreases proportionally to distance from the point force, $Q$ is only proportional to $R$. Thus, it is more effective to be sessile than free-swimming only with a filtration area below a certain size. We denote the critical maximum radius of the filter disk, $R^{*}$. From Eqs. (3) \& (5), this radius can be calculated to be:

$$
R^{*}=3 a / 2
$$

As it is of no consequence whether the force is produced by a flagellum or by other means in the above calculations, the results are valid for any organisms for which the Reynold's number, Re, $\ll 1$. This is the case for most protozoa, and so Eq. (6) could explain, for example, why most suspension-feeding ciliates also attach themselves while feeding. However, care should be taken in applying Eq. (6) too literally, as a number of factors have not been taken into account. The assumption of the organism not having any significant drag, and thus not producing thrust in Case (1), becomes increasingly invalid with increasing cell size. In reality some thrust will always be generated and the total velocity of particles towards the filtration area experienced by the cell will therefore be higher than indicated by Eq. (2), potentially making the value of $R^{*}$ slightly smaller. The exact value of $R^{*}$ depends on a number of factors related to how the force is produced, many of which could also make $R^{*}$ larger under specific circumstances.

In the case of real flagellates, the situation becomes complex since the power needed to propel the spherical cell has been shown theoretically to be only a fraction of the power needed for the flagellum to push itself through the fluid (Brennen 1976, Higdon 1979a,b,c). One could imagine that this would allow for a larger $R^{*}$, but as a large part of the power consumption is due to the added drag of the flagellum which produces thrust, the picture is not clear.

The fact that for flagellates only a small fraction of the total power is used to propel the cell body makes it more valid to neglect the cell body than to neglect the flagellum in theoretical considerations. Lighthill (1976) made an analysis of the motion generated by an infinitely long smooth flagellum. He first calculated the zero-thrust swimming speed: the speed with which the flagellate would swim if it had no hydrodynamical drag to overcome. He then calculated the change in swimming speed and fluid motion that was the result of drag, and thus thrust. In the presence of drag the organism is moving slower than the zero-thrust swimming speed, and so can be said to drift backwards with respect to zero-thrust swimming. Thrust can then be seen as the resistance opposing that backwards drift. In the case of sessile cells attached to particles too large for them to pull, there is no forward motion, and thus compared to the zero-thrust swimming speed, the cells drift backwards with the zero-thrust swimming speed itself. From the resulting thrust, Lighthill calculated the flow through a filtration disk with radius $R$ :

$$
Q=\frac{U R^{2} K_{x} \ln \frac{2 l}{R}}{4 \alpha \eta}
$$

Here $\alpha=N \lambda / L$, where $N$ is the number of waves, $\lambda$ the wavelength and $L$ the total (stretched) length of the flagellum, and $l=N \lambda$, where $l$ is the length of the undulating flagellum. $K_{\mathrm{x}}$ is a crude estimate of a thrust coefficient based on calculations of the resistance coefficients by Gray \& Hancock (1955). It has the form $K_{\mathrm{x}}=$ $2 \pi \eta / \ln (2 q / b)$, where $q$ is a somewhat arbitrary coefficient of a magnitude equal to approximately 0.11 , and $b$ is the radius of the flagellum. From Eqs. (1) \& (7):

$$
R^{*}=21\left(\frac{b}{2 q}\right)^{\frac{21}{L}}
$$

It is seen that $R^{*}$ is a function of $l$, which for a given organism of known flagellar length and diameter is the only variable in the above equation. $1 / L$ is an indication of the length of the flagellum along the axis of the waveform in proportion to its total (stretched) length, and so is inversely related to the amplitude. In Fig. 3, Eq. (8) is plotted for the known parameters of the sessile choanoflagellate Codonosiga sp. with a flagellar

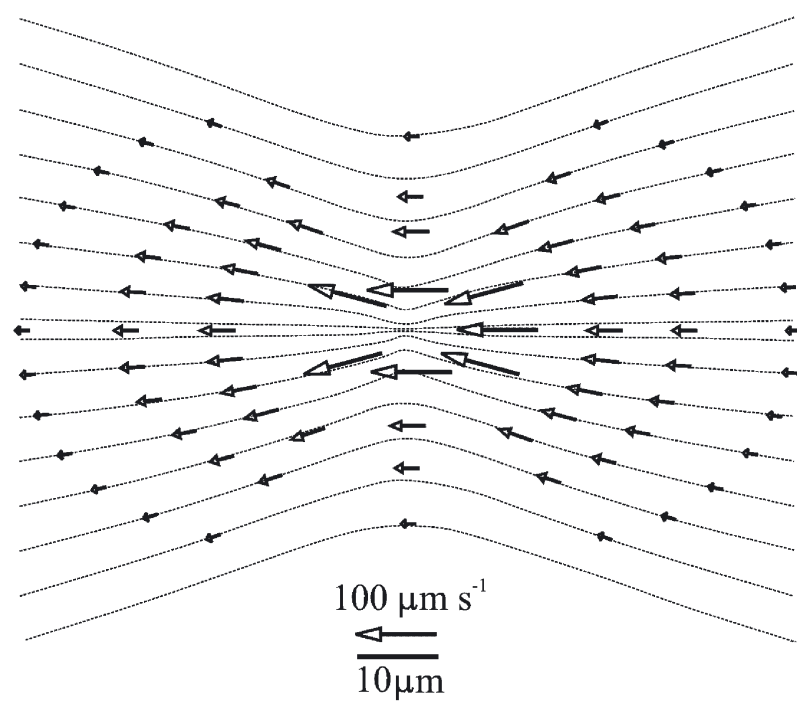

Fig. 2. Stream lines and velocity vectors calculated by singularity analysis for $F=10^{-11} \mathrm{~N}$ as function of distance from a point force 


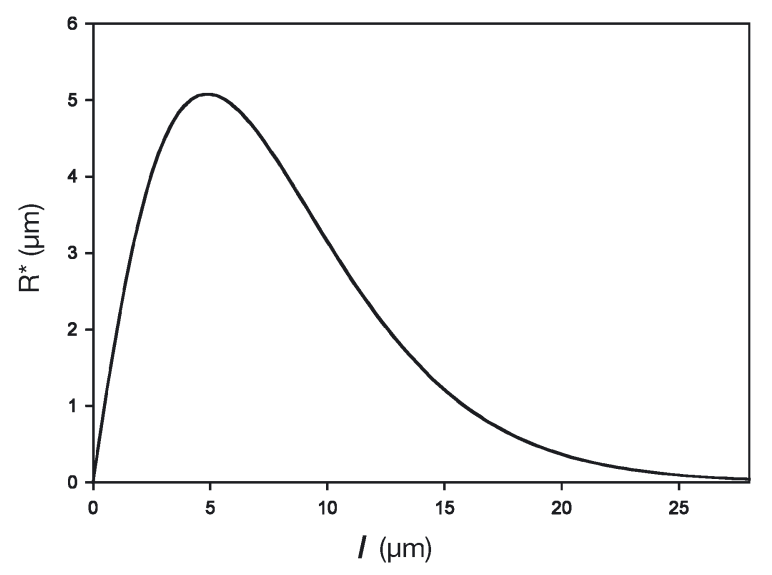

Fig. 3. Calculations based on Lighthill (1976) for the maximum radius of the filtration radius for which attachment is favourable as a function of flagellar length $(l=N \lambda)$. Numerical values are based on the choanoflagellate Codonosiga with a (stretched) flagellum length $L=28 \mu \mathrm{m}$ and a radius of $0.15 \mu \mathrm{m}$. See Table 1 for definitions

length $L$ of ca. $28 \mu \mathrm{m}$ (Sleigh 1964). The figure shows that it is more beneficial for organisms to be sessile when the flagellar waves have large amplitudes. This accords with calculations by Lighthill (1976), showing that for sessile flagellates the most effective amplitude of the flagellar motion is much greater than for freeswimming flagellates. With an estimated filter radius of 5 to $6 \mu \mathrm{m}$, and $l$ ca. $21 \mu \mathrm{m}$ (Sleigh 1964), it would notaccording to Lighthill — be fluid-dynamically beneficial for Codonosiga to be sessile, and so one would assume this to have other causes.

This should not be given too much emphasis, however, since the calculations of Lighthill (1976) were based on the fairly arbitrary variable $q$, and he stated himself that $K_{\mathrm{x}}$ is a very crude estimate. The more accurate slender-body theory, based on calculations of Stokeslets and dipoles along a body with a radius that is extremely small compared to other relevant lengths, was initiated by Hancock (1953). The intense complexity of models of this type made progress impossible without the aid of computers.

Higdon $(1979 a, b, c)$ used the approach to set up systems of singular integral equations to model the swimming motion of whole organisms with smooth flagella moving in a helical (Higdon 1979c) and a planar wave (Higdon 1979a), and the fluid motions generated by planar waves if the organism is sessile (Higdon 1979b). There is no analytical solution to the problems; a complex analytical-numerical approach is required. This makes it impossible to make direct comparisons between the sessile and free-swimming cases without complex computations, although it would be interesting to see the result of such calculations. However, these accurate calculations can be used to test the validity of Lighthill's (1976) model. The numerical results obtained generally showed that Lighthill underestimated swimming speed by ca. 20 to $25 \%$, potentially making the values of $R^{*}$ greater than those shown in Eq. (8). The fact that the force is not a point force, but laterally distributed over an area equivalent to the flagellar amplitude, could also increase $R^{*}$. However, the tendencies of his calculations were correct. It is predicted that the optimal flagellar amplitude for sessile flagellates is about the size of the filtration disk (Higdon 1979b). It is interesting to note that the flagellar amplitudes of sessile choanoflagellates are indeed very large and in accordance with what could be expected from the theoretical models (Higdon 1979b, T. Fenchel pers. obs.).

\section{Experimental results}

Attachment to cover slip

Almost all cells taken from growing Paraphysomonas vestita cultures attached to surfaces in the O-ring chamber within 15 to $20 \mathrm{~min}$ ( $\mathrm{T}_{1 / 2}=3$ to $6 \mathrm{~min}$ ), as illustrated in Fig. 4. Attachment took place under all tested circumstances. Starved cells in particle-free water attached with lower efficiency, but this was not critically tested. Similar experiments were not performed on Pteridomonas danica. (A close relative, Actinomonas mirabilis, attaches only in the presence of bacteria in the water or on bacteria-coated slides. Under such circumstances 72 to $92 \%$ of cells attach with $\mathrm{T}_{1} \frac{1}{2}=2$ to $5 \mathrm{~min}$; $\mathrm{T}$. Fenchel pers. obs.)

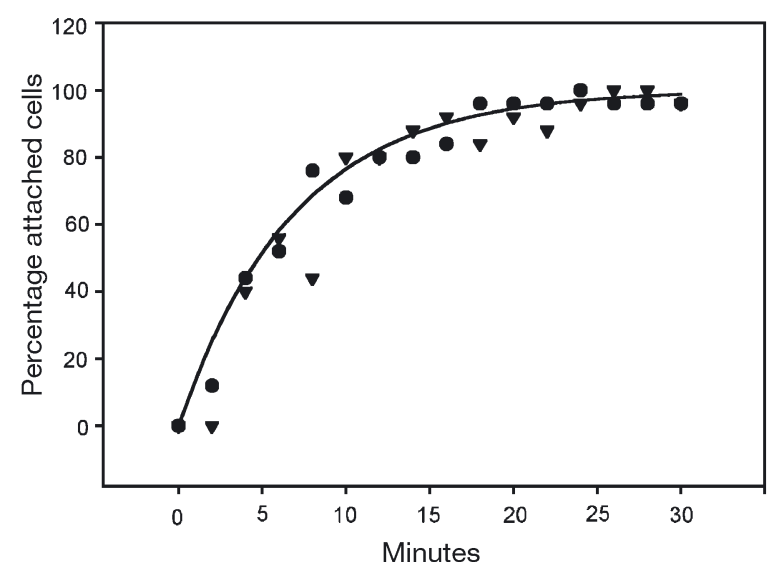

Fig. 4. Attachment of Paraphysomonas vestita to the walls of an O-ring chamber (2 replicate experiments were done as indicated by the 2 different symbols). Data are fitted to $n(t)=$ $100[1-\exp (-\alpha t)]$, with $\alpha$ (probability of a flagellate to attach per unit time) $=0.145 \mathrm{~min}^{-1}$. See Table 1 for definitions 
Feeding currents

For swimming cells, trackings were redrawn to show the flow experienced by the cell; examples of these current systems are shown in Fig. 5, with the flagellate

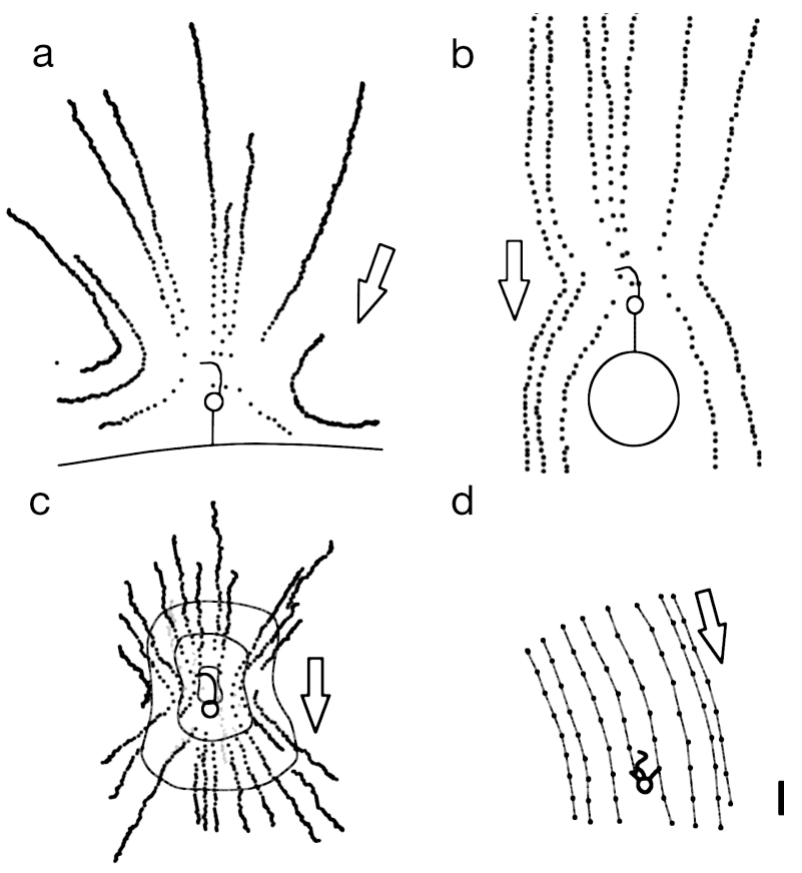

Fig. 5. Flow lines (with the flagellates as reference frame) represented by the position of suspended particles at time intervals of $0.04 \mathrm{~s}$. Arrows indicate the main direction of flow; scale bar $=10 \mu \mathrm{m}$. (a) A Paraphysomonas vestita cell attached to an agar sphere. (b) A P. vestita cell dragging a $25 \mu \mathrm{m}$ latex bead. (c) A $P$. vestita cell attached to a microscope slide drawing water parallel to the slide; also included are isotacs (lines of constant velocity) for the vertical velocity component, representing 100, 40, and $20 \mu \mathrm{m} \mathrm{s} \mathrm{s}^{-1}$. (d) A free-swimming Pteridomonas danica cell

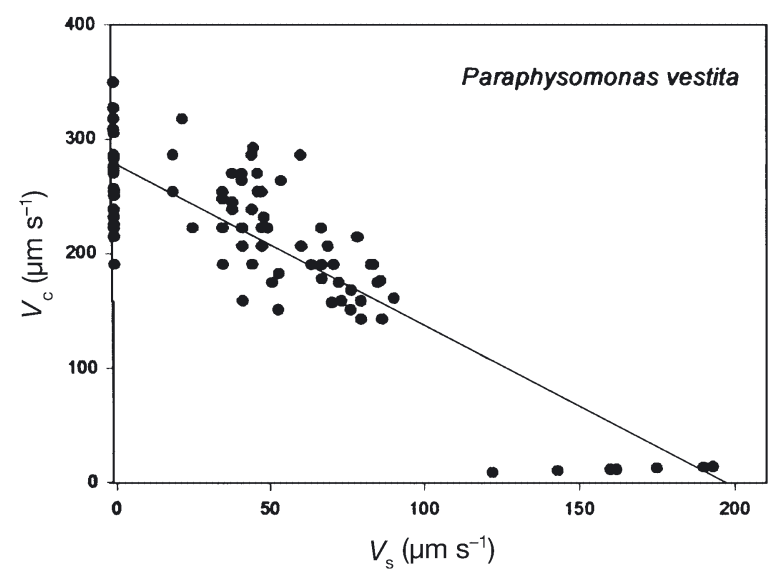

as a reference frame. Free-swimming flagellates experience parallel flow lines with the same speed, but opposite direction of the swimming velocity and only insignificant water currents are generated as compared to the swimming velocity. Cells that attach to particles that they are able to pull forward as they swim simultaneously pull a current towards the cell body, balancing the drag caused by the particle.

The current velocity through the filtration disk caused by swimming is denoted $V_{\mathrm{s}}$. The velocity of the current generated by the thrust of the flagellum was measured within the area perpendicular to the flow within which food particles are intercepted, and at a distance of 1 to 2 cell diameters ahead of the cell. This is denoted $V_{\mathrm{c}}$. The total velocity $V_{\mathrm{t}}$ with which water moves towards the cell is the sum of the velocity at which the cell swims and the velocity with which water is pulled towards the filtration disk, so that $V_{\mathrm{t}}=V_{\mathrm{s}}+V_{\mathrm{c}}$. If the cell is attached to a particle that is too large for it to pull, $V_{\mathrm{s}}=0$, then $V_{\mathrm{t}}=V_{\mathrm{c}}$. Measurements $V_{c}$ and $V_{s}\left(\mu \mathrm{m} \mathrm{s}{ }^{-1}\right)$ were all done on recordings (Fig. 6). When measuring $V_{\mathrm{C}}$ it was often not possible to decide the exact size of the filter of Pteridomonas danica cells. In these cases, the radius of the cells was measured and estimates of the radius of the filtration area estimated as 2a. Paraphysomonas vestita does not have a filter; food particles must make contact with the flagellum, which fans them to the appropriate area of the cell surface (Christensen-Dalsgaard \& Fenchel unpubl.). The effective area of the fluid flow cleared by the flagellum was estimated to be equivalent to the radius of the cell, a (for food particles with a mean radius of $0.75 \mu \mathrm{m})$. Clearance $C\left(\mathrm{ml} \mathrm{h}^{-1}\right)$, and thus filtration efficiency, is directly proportional to $V_{\mathrm{t}}$ :

$$
V_{\mathrm{t}} \pi R^{2} \times 3.6 \times 10^{-9}
$$

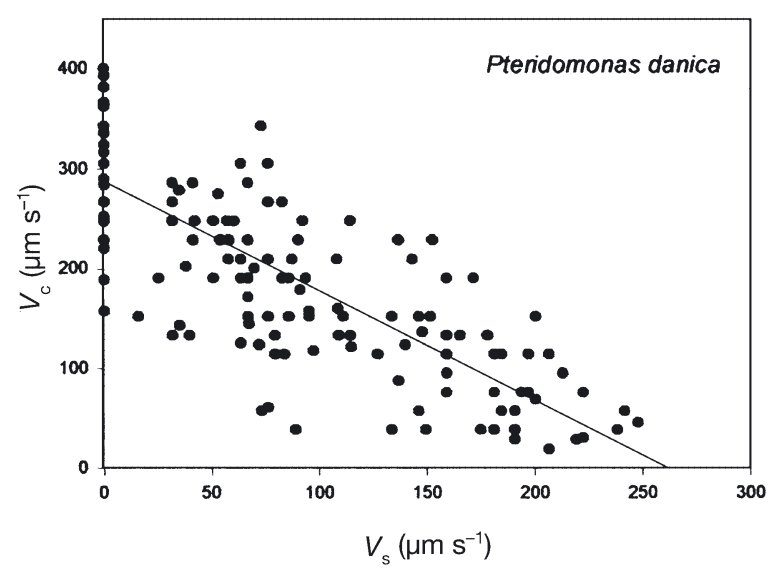

Fig. 6. Paraphysomonas vestita and Pteridomonas danica. Velocity of induced flow $\left(V_{\mathrm{c}}\right)$ as function of swimming velocity $\left(V_{\mathrm{s}}\right)$. Regression lines for the data are also shown 
$V_{\mathrm{t}}$ is plotted as function of swimming speed in Fig. 7. Since both $V_{\mathrm{c}}$ and $V_{\mathrm{s}}$ are directly proportional to force, it can be seen that the proportion of the force $F_{\text {th }}$ used to generate thrust is proportional to $V_{\mathrm{c}} / V_{\mathrm{t}}$, and so $V_{\mathrm{c}} / V_{\mathrm{t}}$ was used as a measure of $F_{\text {th }}$. To clarify tendencies, the average $V_{\mathrm{t}}$ was taken from measurements at intervals of 0.1 for $V_{\mathrm{c}} / V_{\mathrm{t}}$. The average velocity for Paraphysomonas vestita cells generating currents parallel to the surface of the microscope slide was around $125 \mu \mathrm{m} \mathrm{s}^{-1}$, a value well in accordance with the $120 \mathrm{\mu m} \mathrm{s}^{-1}$ measured by Fenchel (1986b). This value is much smaller than the approximate $265 \mu \mathrm{m} \mathrm{s}^{-1}$ observed for $P$. vestita generating currents normal to the surface of agar balls. This difference is due to wall effects, and since the generation of currents parallel to a smooth surface is a situation that would rarely arise in nature, the calculations on sessile cells are based solely on recordings made of cells attached to agar balls. All the average values for $V_{\mathrm{t}}$ were plotted as a function of $V_{\mathrm{c}} / V_{\mathrm{t}}$ from 0 (free-swimming) to 1 (stationary cells). From these values it can be seen that for freeswimming $P$. vestita, $V_{\mathrm{t}}$ is $176 \mu \mathrm{m} \mathrm{s}^{-1}$ and this increases to ca. $300 \mu \mathrm{m} \mathrm{s}^{-1}$ for cells attached to very large particles, or an increase of ca. $70 \%$. For attached Pteridomonas danica the increase in clerance is ca. $34 \%$ relative to freeswimming cells. The values of $R$ are 5 and $2.5 \mu \mathrm{m}$ for $P$. danica and $P$. vestita, respectively. The clearance for freeswimming and attached $P$. danica is $6.39 \times 10^{-5}$ and 8.62 $\times 10^{-5} \mathrm{ml}^{-1} \mathrm{~h}^{-1}$, respectively and for $P$. vestita the corresponding values are $1.24 \times 10^{-5}$ and $2.12 \times 10^{-5} \mathrm{ml}^{-1} \mathrm{~h}^{-1}$.

\section{Estimates from force calculations}

The recordings of Paraphysomonas vestita pulling $25 \mu \mathrm{m}$ spheres showed a mean $V_{\mathrm{s}}$ value of $35 \pm 6 \mu \mathrm{m}$ $\mathrm{s}^{-1}$. From this, and Eq. (2), assuming the diameter of the cell to be $5 \mu \mathrm{m}$, the force generated by the flagellum was calculated to be $F_{f}=9.9 \pm 1.6 \times 10^{-12} \mathrm{~N}$, with a minimum of $7.6 \times 10^{-12} \mathrm{~N}$, and a maximum of $13.0 \times 10^{-12}$ N. Using Eqs. (4) \& (5), and assuming an effective radius of the filtration disk of $2.5 \mu \mathrm{m}$, this results in a theoretical average velocity through the filter $V_{\mathrm{t}}$ of $315.0 \pm 49.5 \mu \mathrm{m} \mathrm{s}^{-1}$, if the force had been used only in producing thrust. For comparison with the experimental results, this calculated value of $V_{\mathrm{t}}$ is plotted in Fig. 7 (open triangle).

To be able to estimate whether wall effects were of importance to the filtering efficiency of attached cells, the length of the attachment thread of Paraphysomonas vestita was measured as distance from cell to sphere on cells pulling the $25 \mu \mathrm{m}$ spheres horizontally. It was found to be $8.2 \pm 2.5 \mu \mathrm{m}$, min. $4.4 \mu \mathrm{m}$, and max. $13.2 \mu \mathrm{m}$. The diameter of the $P$. vestita cells ranged between 3 and $8 \mu \mathrm{m}$.

\section{DISCUSSION}

Earlier estimates of maximal clearance based on direct measurements have given values of $1.7 \times 10^{-5} \mathrm{ml}$ $\mathrm{h}^{-1}$ for Paraphysomonas vestita (Fenchel 1982a) and $7.9 \times 10^{-5} \mathrm{ml} \mathrm{h}^{-1}$ for Pteridomonas danica (Fenchel 1986a). These values are close to, but slightly lower than, those found for attached cells in the present study.

With the exception of Paraphysomonas vestita pulling $25 \mu \mathrm{m}$ spheres, it was not possible to determine the length of the attachment thread on the recordings. The attachment thread of Pteridomonas danica can be very long, and it might make up a significant part of the total drag. Also, it was not possible to determine the dimensions of the particles (starch grains) to which
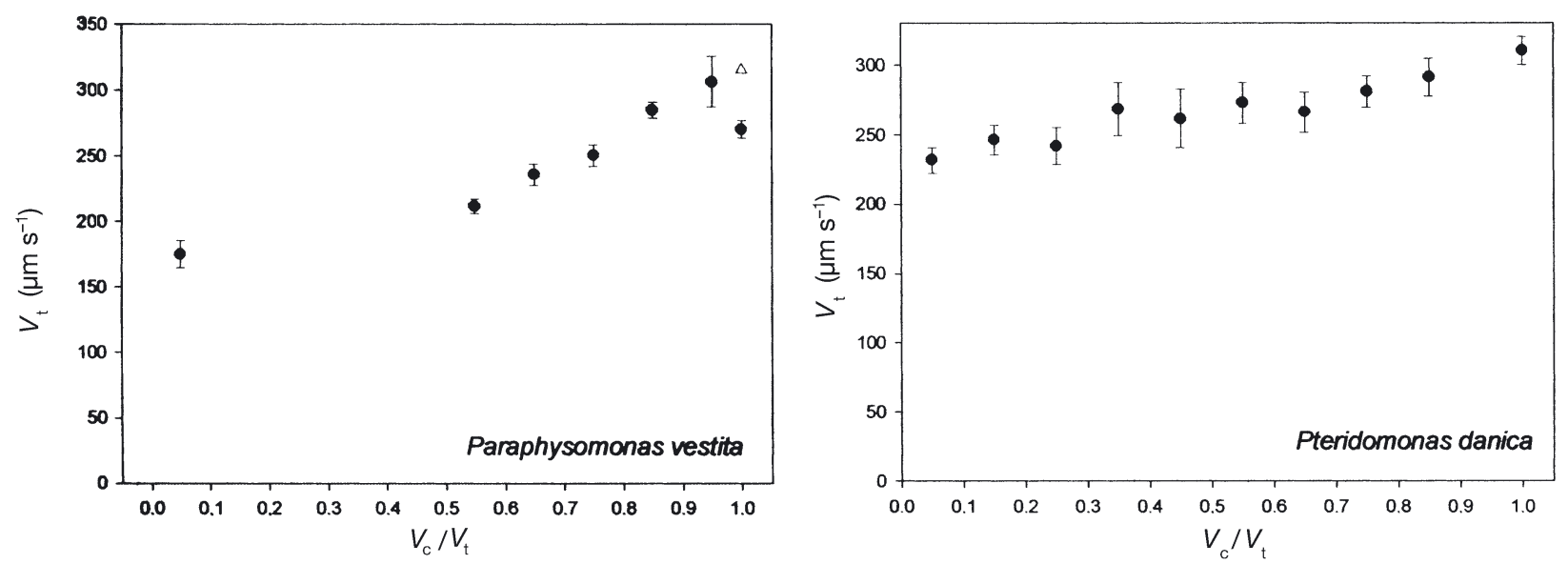

Fig. 7. Paraphysomonas vestita and Pteridomonas danica. Total fluid velocity within the flow area where particles are intercepted $\left(V_{\mathrm{t}}\right)$ as function of the fraction of force for thrust generation $\left(V_{\mathrm{c}} / V_{\mathrm{t}}\right)$. $(\bullet)$ Means $\pm \mathrm{SE}$ for $V_{\mathrm{c}} / V_{\mathrm{t}}$ intervals of 0.1 ; $(\Delta)$ calculated (theoretical) value of $V_{\mathrm{t}}$ for $V_{\mathrm{c}} / V_{\mathrm{t}}=1$ 
the flagellates attached due to glare caused by darkfield illumination, and this rendered it impossible to calculate drag. However, since $V_{\mathrm{c}}$ is directly proportional to thrust, it is seen from the linear relationship between $V_{\mathrm{c}}$ and $V_{\mathrm{s}}$ (Fig. 6) that a large part of the reduction in swimming speed observed was indeed a result of increased drag. The observed scatter could not be caused by cell size since increased cell size would also mean an increase of the thrust, and therefore simply displace the organism along the line. We believe the large deviations are caused by intraspecific variation in force generation. This variation was shown for the calculated force that is generated by the flagella of $P$. vestita; these values vary by a factor of 2 . Since the radius of the sphere is much larger than that of the cell, even a variation in cell radius from 1.5 to $4 \mu \mathrm{m}$ would not explain the observed variation, and so it can be concluded that cells differ with respect to the force generated by the flagellum. The scatter in Fig. 6 is somewhat larger for $P$. danica than for $P$. vestita, indicating a larger variation in force of the flagellum for this species.

Paraphysomonas vestita attached under all recorded circumstances, indicating that attachment does not depend on chemotactic responses. This accords with the increase in filtration efficiency experienced by the cell as a function of $V_{\mathrm{c}} / V_{\mathrm{t}}$ (Fig. 7). The average flow velocity was observed to increase as much as $70 \%$ for attached cells so that it is clearly adaptive to attach.

Values of $V_{\mathrm{c}} / V_{\mathrm{t}}$ from 0.1 to 0.5 are absent for Paraphysomonas vestita in Fig. 7 . This was because the cells were either entirely free-swimming or attached to particles above some minimum size. Thus it was not possible to investigate cells attached to very small particles. Possibly this is because the cells are only freeswimming when dispersing from one attachment site to another, and only remain attached to particles large enough to produce a significant thrust when feeding.

It is interesting to note that the highest feeding flow is not observed for sessile cells attached to the surface of agar spheres, but for cells attached to the largest particles that are still small enough for the cells to be able to pull them (diameter ca. $25 \mu \mathrm{m}$ ). This is judged to be a result of wall effects caused by the proximity of the surface of the agar sphere. Calculations can be done on the flow through a circular filter level with the point force of a Stokeslet as a function of the distance $D$ from the filter to a solid surface (Fenchel 1986b). Using the measured length of the attachment thread of $8.2 \mu \mathrm{m}$, assuming a cell diameter of $5 \mu \mathrm{m}$, and the centre of force of the flagellum to be placed ca. $4 \mu \mathrm{m}$ above the cell surface, the distance between the point force and the attachment surface can be estimated as $D=$ $17 \mu \mathrm{m}$. With radius of the filtration disk $R=2.5 \mu \mathrm{m}, D / R$ $=6.8$. From the model by Fenchel (1986b), the reduc- tion of flow as a result of a presence of a surface at this relative distance should be ca. $10 \%$, which, within the standard errors, explains the observed decrease. The wall effects being a reason for the decrease is further supported by the fact that the calculated value of $V_{\mathrm{t}}$ for an attached cell is somewhat higher than the observed value and in accordance with the general trend observed for Paraphysomonas vestita.

Pteridomonas danica did not show as high an increase in $V_{\mathrm{t}}$ as Paraphysomonas vestita when being attached; the maximal average increase in velocity was ca. $34 \%$. It is consistent with the theoretical considerations that the relative gain in clearance by attachment is higher for $P$. vestita than for $P$. danica because the latter has a much larger filtration area relative to cell radius. The estimate of the effect of attachment in $P$. danica is less certain due to the great variations seen for this organism in $V_{\mathrm{t}}$ for any given value of $V_{\mathrm{c}} / V_{\mathrm{t}}$. As $R>3 a / 2$ for $P$. danica, it is interesting to see that there still seems to be an increase in filtration efficiency, though relatively small. This shows the uncertainty in the theoretical determination of $R^{*}$ by simplified assumptions, as calculated in Eq. (6).

It is likely that the theoretical results for smooth flagella, that is, that the flagellar parameters are important in terms of relative advantage of attachment, also hold for hispid flagella, although the calculations and thus probably the exact tendencies would be different. The differences seen with respect to the fluid-dynamic response of Paraphysomonas vestita and Pteridomonas danica to attachment (Fig. 7) may also be related to the fact that the motility patterns of the flagellum are different in the 2 species (Christensen-Dalsgaard \& Fenchel unpubl.).

Fig. 5 shows that in the presence of the nearby surface, perpendicular to the flow, the flow becomes deformed by the formation of viscous eddies, an effect that is often observed for flows in close proximity to boundaries (e.g. Liron \& Blake 1981). Wall effects also reduce the velocities along the axis of the cell. The velocities are not reduced as much laterally, probably because the flagellum is not a point force; rather, forces act over a larger area proportional to flagellar amplitude and curvature. This could result in higher velocities further from the centre of the force than those predicted theoretically, which could oppose the reduction in velocity from wall effects. In other cases, good agreement between the theoretical (Fig. 2) and observed flow patterns (Fig. 5) can be seen. The observed flow almost has a fore-aft symmetry, indicating that the cell body does not interfere significantly with the flow. This again indicates that theoretical considerations based on the Stokeslet analysis are indeed valid to some approximation, in spite of the fact that the flagellum is not a point force. In general, 
it is therefore likely that attachment is advantageous for suspension-feeding organisms with relatively small filters, because this generates a higher velocity of the fluid through the filter, while using the same force.

Acknowledgements. The study was supported by grants from the Carlsberg Foundation and the Danish Natural Science research Council to T.F.

\section{LITERATURE CITED}

Adler J (1969) Chemoreceptors in bacteria. Science 166: 1588-1597

Alldredge AL, Silver MW (1988) Characteristics, dynamics and significance of marine snow. Prog Oceanogr 20:41-82

Bell W, Mitchell R (1972) Chemotactic and growth responses of marine bacteria to algal extracellular products. Biol Bull 143:265-277

Brennen C (1976) Locomotion of flagellates with mastigonemes. J Mechanochem Cell Motility 3:207-217

Browen JD, Stolzwenbach KD, Chrisholm SW (1993) Simulating bacterial clustering around phytoplankton cells in a turbulent ocean. Limnol Oceanogr 38:36-51

Fenchel T (1982a) Ecology of heterotrophic microflagellates. I. Some important forms and their functional morphology. Mar Ecol Prog Ser 8:211-223

Fenchel T (1982b) Ecology of heterotrophic microflagellates. II. Bioenergetics and growth. Mar Ecol Prog Ser 8:225-231

Fenchel T (1982c) Ecology of heterotrophic microflagellates. IV. Quantitative occurrence and importance as bacterial consumers. Mar Ecol Prog Ser 9:35-42

Fenchel T (1986a) The ecology of heterotrophic microflagel-

Editorial responsibility: John Dolan,

Villefranche-sur-Mer, France lates. In: Marshall KC (ed) Advances in microbial ecology, Vol 9. Plenum Press, New York, p 57-97

Fenchel T (1986b) Protozoan filter feeding. Prog Protistol 1: 65-113

Fenchel T (2001) Eppur si mouve: many water column bacteria are motile. Aquat Microb Ecol 24:197-201

Fenchel T, Blackburn N (1999) Motile chemosensory behaviour of phagotrophic protests: mechanisms for and efficiencies in congregating at food patches. Protists 150: $325-336$

Gray J, Hancock GJ (1955) The propulsion of sea-urchin spermatozoa. J Exp Biol 32:802-814

Hancock GJ (1953) The selfpropulsion of microscopic organisms through liquids. Proc R Soc Lond A 217:96-121

Happel J, Brenner H (1973) Low Reynolds number hydrodynamics. Noordhoff International Publishing, Leyden

Higdon JJL (1979a) A hydrodynamics analysis of flagellar propulsion. J Fluid Mech 90:685-711

Higdon JJL (1979b) The generation of feeding currents by flagellar motions. J Fluid Mech 94:305-330

Higdon JJL (1979c) The hydrodynamics of flagellar propulsion: helical waves. J Fluid Mech 94:331-351

Larsson U, Hagström ^ (1979) Phytoplankton exudates release as an energy source for the growth of pelagic bacteria. Mar Biol 52:199-206

Lighthill J (1975) Mathematical biofluidmechanics. Society for Industrial and Applied Mathematics, Philadelphia

Lighthill J (1976) Flagellar hydrodynamics. Soc Ind Appl Math Rev 18:161-230

Liron N, Blake JR (1981) Existence of viscous eddies near boundaries. J Fluid Mech 107:109-129

Sleigh MA (1964) Flagellar movement of the sessile flagellates Actinomonas, Codonosiga, Monas and Poteriodendron. Q J Microsc Sci 105(4):405-414

Weilbull C (1960) Movement. In: Gunsalus IC, Stainer RY (eds) The Bacteria, I. Academic Press, New York, p 153-205

Submitted: April 2, 2002; Accepted: June 13, 2003

Proofs received from author(s): August 12, 2003 